\title{
A lesson from an old friend: high molecular weight kininogen (HMWK) impact in COVID-19.
}

\author{
Chiara Colarusso $^{1}$, Michela Terlizzi ${ }^{1}$, Aldo Pinto ${ }^{1}$, and Rosalinda Sorrentino ${ }^{1}$ \\ ${ }^{1}$ University of Salerno
}

May 11, 2020

\begin{abstract}
Severe acute respiratory syndrome coronavirus 2 (SARS-CoV2) is a newly identified coronavirus which has spread from China to the rest of the world causing the pandemic coronavirus disease 19 (COVID-19). It has fatality rate that floats from 5 to $15 \%$ and the symtoms are fever, cough, myalgia and/or fatigue up to dyspnea, responsible for hospitalization and in most of the cases of artificial oxygenation. In the attempt to understand how the virus spreads and how to pharmacologically abolish it, it was highlighted that SARS-CoV2 infects human cells by means of angiotensin converting enzyme 2 (ACE2), transmembrane protease serine 2 (TMPRSS2) and 3-chymotrypsin-like protease (3CLpro), also known as Mpro. Once bound to its receptor ACE2, the other two proteases, in concert with the receptor-mediated signaling, allow virus replication and spread throughout the body. Our attention has been focused on the role of ACE2 in that its blockade by the virus increases Bradykinin and its metabolites, well known to facilitate inflammation in the lung (responsible for cough and fever), facilitate both the coagulation and complement system, three mechanisms that are typical of angioedema, cardiovascular dysfunction and sepsis, pathologies which symptoms occur in COVID-19 patients. Thus, we propose to pharmacologically block the kallicrein-kinin system upstream bradykinin and the ensuing inflammation, coagulation and complement activation by means of lanadelumab, which is a clinically approved drug for hereditary angioedema.
\end{abstract}

\section{Abbreviations:}

2'-O-MTase: 2'-O-Ribose Methyltransferase

3CLpro: 3-chymotrypsin-like protease

AAK1: AP2-associated protein kinase 1

ALT: alanine aminotransferase

ARDS: acute respiratory distress syndrome

BKB1R: bradykinin receptor B1

Bradykinin: BK

CFR: case fatality rate

cGAS: cyclic guanosine monophosphate-adenosine monophosphate

cGAMP: cyclic guanosine monophosphate-adenosine monophosphate

$\mathrm{CoV}$ : coronavirus

COVID-19: coronavirus disease 19

CT: computed tomography 
CXCL1: C-X-C motif chemokine 1

CXCL5: C-X-C motif chemokine 5

DABK: des-Arg9bradykinin

DMARDS: disease-modifying antirheumatic drugs

EMA: European medical agency

eNOS: endothelial NOS

HIV: Human Immuno-deficiency Virus

HMWK: high molecular weight kininogen

hrsACE2: human recombinant soluble ACE2

ICU: intensive care unit

iNOS: inducible NOS

INSTI: integrase strand transfer inhibitors

KC: keratinocyte-derived chemokine

KKS: kallikrein-kinin system

LMWK: low-molecular-weight kininogen

Lys-BK: lysyl-bradykinin

mAbs: monoclonal antibodies

MERS: Middle East Respiratory Syndrome

MERS-CoV: Middle East Respiratory Syndrome-CoV

MHC: major histocompatibility complex

MIP2: macrophage inflammatory protein-2

NLRP3: Nod-like receptor protein 3

PAI-1: plasminogen activator inhibitor 1

PAR: protease-activated receptors

PD: peptidase domain

PGI2: prostaglandin I2

RAS: renin-angiotensin system

RDB: receptor binding domain

RdRp: RNA-dependent RNA polymerase

S: Spike

SARS: Severe Acute Respiratory Syndrome

SARS-CoV: Severe Acute Respiratory Syndrome-CoV

SARS-CoV2: Severe acute respiratory syndrome coronavirus 2

scRNA-seq: single-cell RNA sequencing 
STING: stimulator of interferon genes

TF: tissue factor

TLR: Toll-like receptor

TMA: thrombotic microangiopathy

TMPRSS2: transmembrane protease serine 2

u-PA: urokinase-type plasminogen activator

WHO: World Health Organization

\section{Introduction}

Severe acute respiratory syndrome coronavirus 2 (SARS-CoV2) is a newly identified coronavirus which emerged for the first time in the city of Wuhan and rapidly spread through China to cause a disease known as coronavirus disease 19 (COVID-19) (http://www.who.int/csr/don/12-january-2020-novelcoronavirus-china/en/). Because the outbreak of COVID-19 has rapidly spread worldwide, affecting millions of people, the World Health Organization (WHO) has declared SARS-CoV2 as a global pandemic (https://www.who.int/dg/speeches/detail/who-director-general-s-opening-remarks-at-the-mediabriefing-on-covid-19-11-march-2020).

SARS-CoV2 is a new beta-coronavirus belonging to the same sub-group as Severe Acute Respiratory Syndrome-CoV (SARS-CoV) and the Middle East Respiratory Syndrome-CoV (MERS-CoV) which caused SARS and MERS outbreak in 2002 and 2012, respectively (Chen, Liu and Guo, 2020). Several studies have identified a sequence homology of $79.5 \%$ between SARS-CoV2 and SARS-CoV (Zhou et al . 2020b; Wu et al . 2020). Therefore, SARS-CoV2 genome sequencing was rapidly performed, leading to the rapid availability of real-time PCR diagnostic test which is actually used to identify infected subjects allowing the epidemiologic tracking (Corman et al . 2020). SARS-CoV2 is a single-stranded RNA virus characterized by an envelope-anchored Spike glycoprotein which drives virus entry into target cells by binding membrane receptors of sensitive cells and leading to viral replication (Xu et al . 2020b).

Epidemiological data indicate that SARS-CoV2 infection progresses through human-to-human contact, which is predominantly realized via droplet transmission (Ong et al . 2020). As reported by WHO, the incubation period for SARS-CoV2 is 2-14 days, although a longer period may be at the basis of asymptomatic and subclinical infection (https://www.who.int/docs/default-source/coronaviruse/who-china-joint-missionon-covid-19-final-report.pdf ), whereas illness establishment occurs mainly in 10 days (Guan et al . 2020). Although the estimated case fatality rate (CFR) of COVID-19 floats from 5 to $15 \%$, the number of deaths is very high. Indeed, as of May 4th 2020, the virus has infected over 3.4 million individuals in 215 countries, and 238198 people have lost their lives (https://www.who.int/emergencies/diseases/novel-coronavirus-2019).

Several reports have summarized the clinical and epidemiological features of patients affected by COVID-19. In the first published cohort of 41 laboratory-confirmed cases infected with SARS-CoV-2 (Huanget al . 2020), it was reported that infected patients had a median age of 49.0 years and $73 \%$ of them were men. The common symptoms were fever $(98 \%)$, cough $(76 \%)$, myalgia and/or fatigue (44\%), and dyspnea occurred within 8 days from the establishment of the pathology in $55 \%$ of patients. Very few COVID-19 patients had gastrointestinal symptoms and prominent upper respiratory tract signs and symptoms, indicating that the target cells might be located in the upper and lower airways. All hospitalized patients showed abnormalities in chest computed tomography (CT) images, which were characterized by grinding glass-like and consolidation areas in $98 \%$ of the cases reporting bilateral lungs impairment at the basis of bilateral interstitial pneumonia. Because of complications, $32 \%$ of patients were admitted to an intensive care unit (ICU), among which $15 \%$ worsened in a short period of time. Most of them died of respiratory failure, but it is not excluded that death was due to organ failure, coagulation alteration with ensuing thrombosis and embolism as a consequence of blood clotting due to septic shock and/or cardiovascular complications (Huang et al . 2020). An exacerbation 
of SARS-CoV2-induced acute respiratory distress syndrome (ARDS) is characterized by thrombosis and ischemic events so that the check for coagulation parameters are daily needed.

It is well-known that in the early stages of ARDS, fluid from the pulmonary capillaries start to leak into the lungs, making oxygenation very difficult (https://www.lung.org/lung-health-diseases/lung-diseaselookup/ards/learn-about-ards). CT images from COVID-19 patients showed the presence of widespread ground-glass opacities in the lung, which represent the most common evidence of pulmonary edema (Hewitt et al . 2014) associated to bilateral diffuse alveolar damage due to high levels of pro-inflammatory concentrates typical of ARDS, as revealed by biopsy lung specimens from COVID-19 affected patients (Xu et al . 2020d).

\section{Biological targets for SARS-CoV2}

One key discovery in understanding the secrets of SARS-CoV2 infection involves virus structure, especially viral spike (S) protein, which facilitates viral entry into target cells by binding host-cell receptor and then by fusing viral and host membranes (Li, 2016). SARS-CoV2 specifically recognizes Angiotensin-Converting Enzyme 2 (ACE2) as the receptor binding domain (RDB) for its S protein to mediate viral entry and infection (Zhou et al . 2020b). Based on the fact that SARS-CoV2 engages the same receptor used by SARS-CoV to mediate infection, and that both virus share sequence similarities of $80 \%$ between their S proteins (Zhou et al . 2020b), it was suggested that they could act in a similar way. It has to be pointed out that the viral attachment to host cell membrane via ACE2 is the first of a multi-step process involved in coronavirus infection; indeed, after the ligation to ACE2, indispensable for infection, the next step is the priming of S protein by cellular proteases, which consists of S protein cleavage at the S1/S2 and the S2 site, which allows fusion of viral and cellular membranes proteases on the host cell (Letko et al . 2020; Chen et al . 2020). As in the case of SARS-CoV ( $\mathrm{Li}$ et al . 2005a), the $\mathrm{S} 1$ subunit, which contains the RBD, directly interacts with the peptidase domain (PD) of ACE2 providing for tight and higher affinity binding between virus and the host cell. Based on the fact that RBD of SARS-CoV2 is the critical determinant of viral tropism and infectivity, it was demonstrated that its mutations could alter the affinity to the binding receptor, leading to increased viral load (Ouet al . 2020). In particular, three mutants of SARS-CoV2 RBD (V367F, W436R, and D364Y) are correlated to higher human ACE2 affinity, ensuing higher infectivity. This discovery provides insights into SARS-CoV2 evolution and highlights how an increased affinity for human ACE2 due to RDB mutations could further favor COVID-19 diffusion. Because ACE2 is the receptor that SARS-CoV2 uses to anchor host cell, it is obvious to speculate that its expression could be correlated to viral infection susceptibility. Therefore, scientific efforts are focused on the study of ACE2 localization, in order to identify the possible route of viral infection, spread and replication throughout the body. ACE2 expression in the lung is concentrated in a small population of type II pneumocytes, which also express other genes positively correlated to SARS-CoV2 reproduction and transmission (Zhao et al . 2020), suggesting that alveolar pneumocytes could be a potential site of entrance of this virus, and prove a possible explanation for rapid lung viral expansion and pulmonary manifestations typical of COVID-19 patients. If on one side, ACE2-expressing lung cells may be the main target cells for coronavirus infection, on the other, Hamming et al . 2004 have already reported that other organs express ACE2, maybe explaining why some COVID-19 patients also exhibit non-respiratory symptoms. According to the single-cell RNA sequencing (scRNA-seq) and protein datasets, apart from lung and type II alveolar cells, heart, esophagus, kidney, bladder, ileum, oral cavity and testes are the organs at risk due to higher ACE2 expression (Zou et al . 2020; Xuet al . 2020a). To date, in the attempt to find a potential drug against COVID-19, human recombinant soluble ACE2 (hrsACE2), which has already been tested in phase 1 and phase 2 clinical trials for ARDS and COVID-19 (Haschke et al . 2013; Khan et al . 2017; https://clinicaltrials.gov/ct2/show/NCT00886353), can reduce viral growth in Vero E6 cells, most probably by acting as a decoy receptor and preventing viral binding to the natural membrane-bound ACE2 (Monteilet al . 2020).

Beyond ACE2, it was recently found that SARS-CoV2 also uses the cellular transmembrane protease serine 2 (TMPRSS2) for S protein priming, another key event for virus entrance into host cell (Hoffmann et al., 2020). TMPRSS2 is a cell surface protein of the serine protease transmembrane family type II that is 
broadly expressed by epithelial cells (Zouet al . 2020; Xu et al . 2020a) and is involved in the cleavage of the SARS-CoV and influenza virus hemagglutinin protein (Böttcher et al . 2006). As other member of its family, TMPRSS2 favors the entry of the virus into the lungs leading to respiratory infections (Shulla et al . 2011). This protease was already described by Gierer et al . (2013) and Matsuyama et al . (2010) as the enzyme responsible for SARS-CoV infection. Hoffman et al., (2020) found that, SARS-CoV2 uses both TMPRSS2 and endosomal cysteine proteases cathepsin B and L (CatB/L) to enter host cells. The inhibition of TMPRSS2 by means of Camostat mesylate, an TMPRSS2 inhibitor, partially blocked SARSCoV2 entry, suggesting CatB/L involvement (Kawaseet al. 2012). Moreover, the same authors found that co-treatment with Camostat mesylate and E-64d, an inhibitor of CatB/L, completely abrogated virus entry in the same cells, indicating that the virus can use both CatB/L as well as TMPRSS2 for S protein priming in these cell lines. In contrast, the sole Camostat mesylate was not able to block SARS-CoV-2 entry into the TMPRSS2 knock-down 293T cells, confirming that the S protein of SARS-CoV-2 could employ TMPRSS2 for its priming.

Other lines of research are focusing their attention on the coronavirus 3-chymotrypsin-like protease (3CLpro), also known as Mpro, a cysteine protease present in the Coronavirus replicase polyprotein (Zhou et al . 2019). This protease plays a critical role both in the immune regulation and in viral replication in that it regulates the proteolytic cleavage of some polyprotein. 3CLpro drives the cleavage of polyproteins ppla and pplab, which in turn are responsible for the generation of functional proteins such as RNA polymerase, endoribonuclease and exoribonuclease (Khan et al . 2020). For this reason, it was speculated that 3CLpro could represent an attractive target for COVID-19 treatment. In this context, two different molecular docking and molecular dynamic simulation studies reveled 4 drugs that could act against 3CLpro: the antibacterial drug talampicillin, the antipsychotic drug lurasidone (Elmezayen et al. 2020), and the antiviral drug raltegravir and paritaprevir, which were already used in the antiretroviral therapy against the Human Immuno-deficiency Virus (HIV) infections as integrase strand transfer inhibitors (INSTI) (Khanet al . 2020). 3CLpro also cleaves the 2'-O-Ribose Methyltransferase (2'-O-MTase), a protein that catalyzes the methylation of 5'-terminal cap structure of viral mRNAs (Chen et al . 2011). Because this reaction is crucial for viral replication and expression in host cells (Menachery et al . 2014), 2'-OMTase was suggested as another possible druggable target for COVID-19 treatment (Khan et al . 2020), although it is still unclear whether 2'-O-MTase, as well as 3CLpro, contributes to SARS-CoV2 infection.

\section{ACE2 and Bradykinin}

ACE2 is a membrane-associated aminopeptidase and belongs to the angiotensin-converting enzyme family of dipeptidyl carboxydipeptidases and has high homology to human angiotensin 1 converting enzyme (ACE1) (Tipnis et al . 2000). A region of the extracellular portion of ACE2 that includes the first $\alpha$-helix and lysine 353 and proximal residues of the $\mathrm{N}$ terminus of $\beta$-sheet 5 interacts with high affinity to the receptor binding domain of the SARS-CoV S glycoprotein ( $\mathrm{Li}$ et al . 2005b). The secreted ACE2 catalyzes the cleavage of angiotensin I into angiotensin 1-9, and angiotensin II into the vasodilator angiotensin 1-7, explaining the negative regulation activity exerted on angiotensin II-induced increase of blood pressure (Patel et al - 2016). Beyond its role in the cardiovascular system, it plays a role in the regulation of renal function and fertility (Koitka et al . 2008; Pan et al . 2013). Of recent outbreak, it was demonstrated that ACE2 plays as a functional receptor for the S glycoprotein of the human coronavirus SARS-CoV2 (COVID-19 virus) (Imai et al . 2005; Zhou et al . 2020b). Once the virus binds with its glycoprotein moiety of the extracellular S protein to ACE2, it is endocytosed into the host cell allowing its reproduction, leading to ARDS- and SARS-induced pulmonary edema. The ligation of ACE2 by the virus provides ACE2 blockade which systemically translates into higher hypertensive activity of Angiotensin II, which is not catabolized into angiotensin 1-7 or angiotensin 1-9, promoting not only the well-known hypertensive and hypertrophic activity on the cardiovascular system, but also the leakage of pulmonary blood vessels, as demonstrated in an in vivomodel (Imai et al . 2005) (Figure 1). This likely leads to what we are actually assisting in terms of high blood pressure in COVID-19 patients and pulmonary edema up to angioedema, which underlies the fact that ACE2 cleaves a single-terminal residue of several bioactive peptides, such as neurotensin, dynorphin A (1-13), apelin-13, and des-Arg ${ }^{9}$ bradykinin (DABK) (Vickers et al . 2002; Donoghue et al . 
2003). Herein, once ACE2 is blocked by SARS-CoV2, besides the perturbation of the pulmonary reninangiotensin system (RAS) (Imai et al . 2005; Kuba et al . 2005), increasing inflammation and vascular permeability occur, due to the activity of DABK that binding to bradykinin receptor B1 (BKB1R) can lead to acute lung inflammation (Sodhi et al . 2018) (Figure 1). In support, in a mouse model of endotoxin inhalation, the absence of ACE2 led to the activation of the DABK/BKB1R axis, release of pro-inflammatory chemokines such as C-X-C motif chemokine 5 (CXCL5), macrophage inflammatory protein-2 (MIP2), C-X-C motif chemokine 1 (CXCL1), also known as keratinocyte-derived chemokine (KC), and TNF- $\alpha$ from airway epithelia, increasing neutrophilia and inflammation with an ensuing lung injury. To date, the same authors showed that endotoxin administration in mice induced ACE2 attenuation in the lung partly due to NF- $\varkappa \mathrm{B}$ signaling, which is constantly activated during an acute inflammatory process, especially by IL- $1 \beta$, as well as IL-6, and other pro-inflammatory cytokines, exacerbating lung inflammation/edema up to organ dysfunction (Sodhi et al . 2018).

Therefore, if we consider the clinical outcome of COVID-19 patients, we could speculate that the blockade or under-expression of ACE2 by SARS-CoV2 and the ensuing pro-inflammatory mediators (i.e. IL-1 $\beta$ and IL-6) contribute to the pathogenesis of lung inflammation because of an impaired catabolism of DABK, leading to an enhanced BKB1R signaling, resulting in what is actually called "cytokine storm" during the early onset of COVID-19. Bradykinin receptors are B1, an induced receptor during inflammatory conditions, and B2, a constitutive and ubiquitous receptor (Marceau, Hess, and Bachvarov, 1998). B2 receptor mediates the action of BK and lysyl-bradykinin (Lys-BK), the first set of bioactive kinins formed in response to injury from kininogen precursors through the action of plasma and tissue kallikreins; whereas B1 receptor mediates the action of DABK and Lys-DABK, the second set of bioactive kinins formed through the action of carboxypeptidases on BK and Lys-BK, respectively (Couture et al . 2001). The B2 receptor is ubiquitous and constitutively expressed, whereas the B1 receptor is expressed at very low levels in healthy tissues but it is induced following injury by various pro-inflammatory cytokines such as IL-1 $\beta$ (Marceau , Hess, and Bachvarov, 1998). Both receptors act through $\mathrm{G} \alpha_{\mathrm{q}}$ to stimulate phospholipase $\mathrm{C} \beta$ followed by phosphoinositide hydrolysis and intracellular free $\mathrm{Ca}^{2+}$ mobilization, and through $\mathrm{G}_{\mathrm{i}}$ to inhibit adenylate cyclase and stimulate the MAPK pathways (Leeb-Lundberg, 2004) (Figure 2). Although little is known about the cross-talk between B1 and B2 receptors, it is well established that B2 signalling can mediate B1 upregulation via MAPK- and NF-kB-dependent pathways, and that the expression of both receptors can be induced by pro-inflammatory cytokines (i.e. TNF $\alpha$ and IL-1 $\beta$ ), creating a "catch-22 loop" (Brechter et al . 2008). This molecular mechanism/s translated into clinical outcomes underlie vascular permeability and dilation, bronchoconstriction (cough) and pain (hyperalgesia, muscular pain) with ensuing fever due to the cytokine storm, all symptoms of COVID-19. (Figure 1).

An important issue is that BK is produced by the kallikrein-kinin system (KKS). The KKS consists of prekallikrein in complex with high molecular weight kininogen (HMWK) (Hooley, McEwan, and Emsley, 2007) (Figure 2). HMWK is a multifunctional single-chain plasma glycoprotein primarily expressed by the liver and secreted into the bloodstream. HMWK consists of 6 different proteic domains (Shariat-Madar and Schmaier, 1999) and binds to prekallikrein by means of a sequence in domain 6 . The detachment of the domain 4 liberates BK (Griffin and Cochrane, 1979). Kallikreins are serine proteases responsible for the release of kinins, vasoactive peptides that cause vascular smooth muscle relaxation and an increase of vascular permeability (Bhoola, Figueroa, and Worthy, 1992). It has been found that kallikrein exists in two different forms: plasma kallikrein, which cleaves HMWK into BK, which in turn interacts with the constitutive B2 receptor, and tissue kallikrein which processes low-molecular-weight kininogen (LMWK) into Lys-BK. The interaction of BK or Lys-BK onto B1 and B2 receptors will increase the activation of both endothelial NOS (eNOS) and inducible NOS (iNOS), with an ensuing release of nitric oxide, potent vasodilator, of prostaglandin I2 (PGI2) and pro-inflammatory cytokines and chemokines responsible for acute inflammation that is accompanied by vasodilation, pain, cell proliferation and fibrosis (Kuhr et al . 2010; Tsai et al . 2015), symptoms typical of COVID-19 (Figure 1; Figure 2).

Plasma as well as tissue kallikrein are initially secreted as inactive, but both of them are activated by serine protease activity (Bhoola, Figueroa, and Worthy, 1992). The reciprocal activation of Factor XIIa (Hageman 
Factor) and plasma prekallikrein promotes the activation of the kallikrein, which, besides the catabolism of HMWK into BK, initiate the intrinsic pathway of coagulation, influencing fibrinolysis (Figure 2). At the same time, tissue pre-kallikrein cleaves low molecular weight kininogen (LMWK) in des-Arg-kallidin and des-Arg ${ }^{9}$ BK which interact with B1 receptors further enhancing inflammation. The intrinsic pathway of coagulation is then correlated to the extrinsic pathway of the coagulation in that Factor XIIa activates Factor XI, which leads to the activation of factor IX which subsequently leads into the common pathway by the activation of Factor X and then thrombin, with fibrin aggregates generation, hence the need to detect D-dimer in COVID19 patients (Figure 2). In this context, studies looking at rat models that express both BK receptors show, in vitro, that BK acting through the B2 receptor on the surface of endothelial cells promotes the expression of procoagulant and antifibrinolytic proteins, such as tissue factor (TF) and plasminogen activator inhibitor 1 (PAI-1) (Kimura et al . 2002). On the other hand, plasma kallikrein can align pro-urokinase plasminogen activator (u-PA) in such close proximity as to drive plasminogen activation into plasmin which degrades fibrin aggregates (Selvarajan et al . 2001), effects that are widely observed in sepsis, another co-morbidity of COVID-19. However, it has been shown that the complex HMWK and Factor XIIa can also bind to another of the three endothelial cell-binding sites, the 33-kDa cell surface receptor for the first component of complement C1q (gC1qR/p33) which has high affinity for HMWK (Ghebrehiwet et al . 2006). Therefore, the activation of the classical pathway of the complement together with the activation of the plasmin on the conversion of C3 into C3a and C3b induce the activation of both lecithin and extrinsic pathways of the complement with the ensuing activation of the humoral immunity, exacerbating the inflammatory process (Figure 2).

These events may happen in COVID-19 patients from the early onset up to the severe step of the pathology. To date the above pathological conditions are typical of angioedema, cardiovascular dysfunction and sepsis, pathologies which symptoms occur in COVID-19 patients. But it is obvious to ask the correlation between these symptoms and the viral infection. Why would this happen? Our hypothesis is that the infection by SARS-CoV2 that "uses" ACE2 to enter the host, blocks the activity of the degradation of angiotensin II, but at the same time the degradation of BK is altered and impaired in that to trigger and enhance all the above described clinical events.

\section{Approaching therapies for COVID-19 patients.}

In the attempt to identify the effective anti-SARS-CoV2 therapy some clinical trials are still ongoing. In particular, the therapeutic approach can be classified in two big branches: the antiviral, which aims to diminish virus replication, and the anti-inflammatory agents to hijack the cytokine storm that the virus is able to induce. In particular, the antiviral drug that is currently proving of efficacy in COVID-19 is remdesivir, which tightly binds and inhibits the virus RNA-dependent RNA polymerase (RdRp) (Elfiky, 2020). In a cohort of patients hospitalized for severe COVID-19 who were treated with compassionate-use, remdesivir proved of clinical improvement in 36 out of 53 patients (68\%) (Grein et al. 2020). Instead, lopinavir and ritonavir, anti-HIV drugs, showed disappointing results beyond standard care in that the viral load and the mortality were not altered (Caoet al. 2020).

On the other hand, disease-modifying antirheumatic drugs (DMARDS), such as chloroquine and hydroxychloroquine, as well as immunotherapeutic agents, such as monoclonal antibodies (mAbs), are being used. In particular, chloroquine as well as hydroxychloroquine, antimalarial drugs, can interfere with lysosomal activity and autophagy, interact with membrane stability and alter signalling pathways and transcriptional activity, which can result in inhibition of cytokine production and modulation of immune co-stimulatory molecules (Schrezenmeier and Dörner, 2020). Thus, they can inhibit lysosomal activity, preventing major histocompatibility complex (MHC) class II-mediated antigen presentation. Moreover, they can accumulate in endosomes and bind to double-stranded DNA, inhibiting both Toll-like receptor (TLR) signaling (i.e. TLR7 and TLR9) (Kuznik et al.2011) and the nucleic acid sensor cyclic guanosine monophosphate-adenosine monophosphate (cGMP-AMP or cGAMP) synthase (cGAS) (Zhang et al. 2014). By preventing TLR signalling and cGAS-stimulator of interferon genes (STING) signalling, hydroxychloroquine can reduce the production of pro-inflammatory cytokines (van den Borne et al. 1997). However, the adverse effects need to be taken 
into consideration, especially in regards to the alteration of heart rhythm which cautiously limits their use.

Importantly, immunotherapeutic agents, such as tocilizumab or sarilumab, which are mAbs against IL-6 signalling, highly released during the interstitial pneumonia, have proved an effective treatment in severe patients of COVID-19 to calm the inflammatory storm and reduce mortality (Xu et al. 2020c; http://www.news.sanofi.us/2020-03-16-Sanofi-and-Regeneron-begin-global-KevzaraR-sarilumab-clinical-trial-program-in-patients-with-severe-COVID-19). This encouraging clinical trial indicates that neutralizing mAbs against other pro-inflammatory cytokines may also be of use, with potential targets including IL-1, IL-17 and their respective receptors. However, tocilizumab, as well as sarilumab, can induce hepatotoxicity, neutropenia, tumorigenesis, hypersensitivity, opportunistic infections (https://www.accessdata.fda.gov/drugsatfda_docs/label/2016/125276s107_125472s018lbl.pdf; https://www.accessdata.fda.gov/drugsatfda_docs/label/2018/761037s001lbl.pdf); therefore, mAbs targeting IL-6 signalling cannot be administered to all patients, because of co-morbidities to COVID-19 need carefully to be taken into consideration. Nevertheless, they represent the promise for blocking cytokine storm-related immunopathology of moderate to severe COVID-19.

Another clinical trial for stable COVID-19 patients is on the activity of colchicine, an antigout drug, which blocks the mitotic cells in metaphase, but is also able to block Nodlike receptor protein 3 (NLRP3) inflammasome inhibiting the release of IL-1-like cytokines ( $h t t$ ps://clinicaltrials.gov/ct2/show/NCT04322565), such as IL-1 $\beta$, that was in the attempt to be blocked by means of anakinra in another clinical trial (https://clinicaltrials.gov/ct2/show/NCT04366232). Additionally, emapalumab, a monoclonal antibody against IFN- $\gamma$, associated to anakinra, has been proposed (https://clinicaltrials.gov/ct2/show/NCT04324021). However, the inhibition of such an important anti-viral cytokine could be on one side important to block the cytokine storm, but on the other can be hijacked by the virus due to the absence of one of the most important army against viral infections, creating further opportunistic pathologies.

Baricitinib has been identified as a molecule potentially useful in COVID-19 because of a double action to down-modulate the inflammatory storm and reduce the entry of the virus into type II pneumocytes due to the blockade of the AP2-associated protein kinase 1 (AAK1), a regulator of the endocytosis of the virus (Richardson et al. 2020). Moreover, baricitinib also binds to the cyclin G-associated kinase, another regulator of endocytosis. Thus, baricitinib may be useful for both reducing inflammatory response and viral endocytosis.

It has to be pointed out that all the above ongoing clinical trials include monitoring of coagulation parameters, such as D-dimer, which is a metabolite of fibrin aggregates. Although there are no published case series reporting abnormal coagulation parameters in hospitalized severe COVID-19 patients, in a multicenter retrospective cohort study in China, elevated D-dimer levels $(>1 \mathrm{~g} / \mathrm{L})$ were strongly associated with in-hospital deaths, therefore to severe COVID-19 (Zhou et al. 2020a). To date, low molecular weight heparin (LMWH), enoxaparin, has been proposed for these patients either to avoid thromboembolism events (Tang et al. 2020a) or to inhibit the cytokine storm (Shiet al. 2020), due to non-anticoagulant fraction of enoxaparin suppresses in vitro IL-6 and IL-8 release from human pulmonary epithelial cells (Shastri et al. , 2015). Moreover both in vitro and in vivo experimental studies have shown that human coronaviruses utilize heparin sulfate proteoglycans for attachment to target cells (Milewska et al. 2014). Indeed, interaction between the SARS-CoV2 Spike S1 protein receptor binding domain (SARS-CoV-2 S1 RBD) and heparin has been recently showed, suggesting a role for heparin in the therapeutic armamentarium against COVID-19 (Mycroft-West et al. 2020).

Another immunotherapeutic agent that was suggested is eculizumab, a mAb against C5 complement. Diffuse microvascular thrombi in multiple organs in COVID-19 non-survivors have been announced and even more important, thrombotic microangiopathy (TMA) can occur in many different clinical scenarios including pathogenic complement activation (Campbell and Kahwash, 2020). Altered complement system occurs in a number of pathologic settings, leading to diffuse thrombotic microangiopathy (TMA), microangiopathic hemolytic anemia, thrombocytopenia, and acute renal failure up to organ dysfunction. If given early, 
eculizumab therapy can reverse both renal and cardiac dysfunction (Campbell and Kahwash, 2020). Campbell and Kahwash (2020) suggest that complement inhibition could be a promising treatment for severe COVID19 by reducing the innate immune-mediated consequences of severe coronavirus infection, and it would pair well with direct anti-viral therapy.

Another suggested approach includes the off-label use of Camostat or Nafamostat mesylate, inhibitors of the host cell protease TMPRSS2, that could arrest coronavirus infections by controlling viral entry into the human cells. It has to be noted that, if on one side TMPRSS2 inhibitors could prevent SARS-CoV2 replication by blocking the fusion of the virus envelope with host cell surface membranes, they could also be effective in controlling pathological conditions correlated to COVID-19, such as coagulation and inflammation, based on their pharmacological properties. As it is well-known, Nafamostat mesylate has been used as a short-acting anticoagulant in patients with disseminative blood vessel coagulation, hemorrhagic lesions, and hemorrhagic tendencies (Maruyama et al.2011; Choi et al. 2015) due to its ability to competitively inhibit various enzyme systems, such as coagulation and fibrinolytic systems (thrombin, Xa, and XIIa), the KKS, the complement system, pancreatic proteases and activation of protease-activated receptors (PARs) (Kim et al. 2016). Similarly, Gabexate mesylate, binds and inhibits kallikrein, plasmin and thrombin (Tamura et al. 1977). Therefore, it was suggested the use of these drugs to prevent thrombosis and disseminated intravascular coagulation typical of COVID-19 patients (Tang et al. 2020b; Cui et al. 2020). Beyond their anticoagulant propriety, both Gabexate and Nafamostat mesylate show anti-inflammatory effects, which could be useful in COVID-19 uncontrolled inflammation (Tay et al. 2020). In particular, Gabexate mesylate decreases the production of inflammatory cytokines, such as TNF- $\alpha$ by attenuating NF- $\chi \mathrm{B}$ and JNK pathway activity, most probably through the proteolytic destruction of IxB (Yuksel et al. 2003); Nafamostat mesylate shows an anti-inflammatory effectin vitro, where it mediates the inhibition of lipopolysaccharide-induced nitric oxide production, apoptosis, IL-6 and IL-8 production in cell cultures (Kang et al. 2015; Choi et al. 2016). In this context, Camostat mesylate could be also useful to reduce the production of inflammatory cytokines due to SARS-CoV2 infection. Indeed, it was already found that Camostat mesylate reduces the release of IL-6 and TNF- $\alpha$ into cell supernatants infected with influenza virus (Yamaya et al. 2016).

\section{Further therapeutic hypotheses.}

So far, the published clinical observations of biochemical markers in COVID-19 patients include elevated LDH, D-dimer, bilirubin, high levels of pro-inflammatory cytokines that accompany interstitial pneumonia, renal and cardiac injury due to thromboembolic events, which also underlie septic shock that occurs in severe COVID-19 patients. Therefore, based on what described above and cross-linking biochemical with clinical outcomes, in this review we propose another therapeutic approach based on the inhibition of both BK receptors and HMWK. Icatibant is an antagonist of B2 receptor blocking the activity of the BK avoiding both the pro-inflammatory cytokine storm and cell proliferation; it is a drug approved by the European medical agency (EMA) for the treatment of angioedema in both children and adults (https://www.ema.europa.eu/en/documents/assessment-report/firazyr-epar-public-assessmentreport_en.pdf). No specific adverse events have been reported, unless urticaria, nausea and headache, though, specific attention to be paid in patients with compromised cardiovascular system (i.e. ischemia and angina pectoris). However, preclinical studies did not show any genotoxic activity, alteration of the cardiac conduction and ischemia events or hemodynamic parameters. Nevertheless, it has been demonstrated that icatibant highly binds to B2 receptor, while the affinity to the analogous B1 receptor is at least 100 times lower. It has to be pointed out, though, that little is known about B1 receptor, which is the inducible receptor during inflammatory conditions, although several pre-clinical and phase I/II trials have been performed to evaluate possible use of agents targeting B1 receptor for inflammation-related diseases (Qadri and Bader, 2018).

In addition, another drug to point the attention on could be lanadelumab, which is a monoclonal antibody against the plasmatic kallikrein, which is important for the cleavage of HMWK into BK, and is involved in the coagulation as well as in the induction of the complement system (Figure 2). Actually, lanadelumab is used for the treatment of angioedema and has not reported adverse, severe events, other than hypersensitivity, myalgia and hepatic alteration of 
alanine aminotransferase (ALT) (https://www.ema.europa.eu/en/documents/assessment-report/takhzyroepar-public-assessment-report_en.pdf). Differently from icatibant, lanadelumab could block upstream the activity of BK, avoiding the inflammatory and coagulation storm besides the complement system in SARSCoV2 infected patients, likely preventing the exacerbation of COVID-19, in parallel with antiviral therapy.

In conclusion, we believe that the blockade of ACE2 increases not only the activity of angiotensin II on the cardiovascular system, but also the levels of DABK derived by HMWK. Therefore, the hypothesis to block the production of DABK upstream by blocking the metabolism of HMWK could be another option to face this tremendous pandemic event that affected whole world life style obliging to social limitations and stay-at-home politics.

\section{Acknowledgements:}

$\mathrm{N} / \mathrm{A}$

\section{Conflict of Interest:}

No conflict of interest to disclose.

\section{References}

Bhoola KD, Figueroa CD, Worthy K (1992). Bioregulation of kinins: Kallikreins, kininogens, and kininases. Pharmacol Rev 44(1): 1-80.

Böttcher E, Matrosovich T, Beyerle M, Klenk HD, Garten W, Matrosovich M (2006). Proteolytic activation of influenza viruses by serine proteases TMPRSS2 and HAT from human airway epithelium. J Virol 80(19): 9896-9898. https://doi.org/10.1128/JVI.01118-06

Brechter AB, Persson E, Lundgren I, Lerner UH (2008). Kinin B1 and B2 Receptor Expression in Osteoblasts and Fibroblasts Is Enhanced by interleukin-1 and Tumour Necrosis Factor-Alpha. Effects Dependent on Activation of NF-kappaB and MAP Kinases. Bone 43(1): 72-83. doi: 10.1016/j.bone.2008.02.003.

Campbell CM, Kahwash R (2020). Will Complement Inhibition Be the New Target in Treating COVID-19 Related Systemic Thrombosis? Circulation doi: 10.1161/CIRCULATIONAHA.120.047419.

Cao B, Wang Y, Wen D, Liu W, Wang J, Fan G et al (2020). A Trial of Lopinavir-Ritonavir in Adults Hospitalized with Severe Covid-19. N Engl J Med NEJMoa2001282. doi: 10.1056/NEJMoa2001282.

Chen Y, Guo Y, Pan Y, Zhao ZJ (2020). Structure analysis of the receptor binding of 350 2019-nCoV. Biochem Biophys Res Commun pii: S0006-291X(20)30339-9. doi: 10.1016/j.bbrc.2020.02.071.

Chen Y, Liu Q, Guo D (2020). Emerging coronaviruses: genome structure, replication, and pathogenesis. J Med Virol 92: 418-423. https://doi.org/10.1002/jmv.25681.

Chen Y, Su C, Ke M, Jin X, Xu L, Zhang Z et al (2011). Biochemical and structural insights into the mechanisms of SARS coronavirus RNA ribose2'-O-methylation by nsp16/nsp10 protein complex. PLoS Pathog 7(10): e1002294-e1002294. doi: 10.1371/journal.ppat.1002294.

Choi JY, Kang YJ, Jang HM, Jung HY, Cho JH, Park SH et al (2015). Nafamostat Mesilate as an Anticoagulant During Continuous Renal Replacement Therapy in Patients With High Bleeding Risk: A Randomized Clinical Trial. Medicine (Baltimore) 94(52): e2392. doi: 10.1097/MD.0000000000002392

Choi S, Kwon HJ, Song HJ, Choi SW, Nagar H, Piao S et al (2016). Nafamostat mesilate promotes endothelium-dependent vasorelaxation via the Akt-eNOS dependent pathway. Korean J Physiol Pharmacol 20(5): 539-45. doi: 10.4196/kjpp.2016.20.5.539.

Corman VM, Landt O, Kaiser M, Molenkamp R, Meijer A, Chu DKW et al (2020). Detection of 2019 novel coronavirus (2019-nCoV) by real-time RT-PCR. Euro Surveill 25(3): 2000045. doi: 10.2807/15607917.ES.2020.25.3.2000045 
Couture R, Harrisson M, Vianna RM, Cloutier F (2001). Kinin receptors in pain and inflammation. Eur J Pharmacol 429: 161-176. doi: 10.1016/s0014-2999(01)01318-8.

Cui S, Chen S, Li X, Liu S, Wang F (2020). Prevalence of venous thromboembolism in patients with severe novel coronavirus pneumonia. J Thromb Haemost. doi:10.1111/jth.14830

Donoghue M, Wakimoto H, Maguire CT, Acton S, Hales P, Stagliano N et al (2003). Heart block, ventricular tachycardia, and sudden death in ACE2 transgenic mice with downregulated connexins. J Mol Cell Cardiol 35: 1043-1053. doi:10.1016/S0022-2828(03)00177-9.

Elfiky AA (2020). Ribavirin, Remdesivir, Sofosbuvir, Galidesivir, and Tenofovir against SARS-CoV2 RNA dependent RNA polymerase (RdRp): A molecular docking study. Life Sci 253: 117592. doi: $10.1016 /$ j.lfs.2020.117592

Elmezayen AD, Al-Obaidi A, Şahin AT, Yelekçi K (2020). Drug repurposing for coronavirus (COVID-19): in silico screening of known drugs against coronavirus 3CL hydrolase and protease enzymes. J Biomol Struct Dyn 1-12. doi: 10.1080/07391102.2020.1758791

Ghebrehiwet B, CebadaMora C, Tantral L, Jesty J, Peerschke EI (2006). gC1qR/p33 serves as a molecular bridge between the complement and contact activation systems and is an important catalyst in inflammation. ADV EXP MED BIOL 586: 95-105. doi: 10.1007/0-387-34134-x_7 PMID: 16893067

Gierer S, Bertram S, Kaup F, Wrensch F, Heurich A, Krämer-Kühl A et al (2013). The spike protein of the emerging betacoronavirus EMC uses a novel coronavirus receptor for entry, can be activated by TMPRSS2, and is targeted by neutralizing antibodies. J Virol 87(10): 5502-5511. https://doi.org/10.1128/JVI.00128-13

Grein J, Ohmagari N, Shin D, Diaz G, Asperges E, Castagna A et al (2020). Compassionate Use of Remdesivir for Patients With Severe Covid-19. N Engl J Med NEJMoa2007016. doi: 10.1056/NEJMoa2007016

Griffin JH, Cochrane CG (1979). Recent advances in the understanding of contact activation reactions. Semin Thromb Hemost 5(4): 254-73. doi: 10.1055/s-0028-1087158

Guan W, Ni Z, Hu Y, Liang W, Ou C, He J et al (2020). Clinical characteristics of coronavirus disease 2019 in China. N Engl J Med 382: 1708-1720. doi: 10.1056/NEJMoa2002032

Hamming I, Timens W, Bulthuis MLC, Lely AT, Navis G, van Gooret H (2004). Tissue distribution of ACE2 protein, the functional receptor for SARS coronavirus. A first step in understanding SARS pathogenesis. Journal of Pathology 203: 631-637. doi: 10.1002/path.1570

Haschke M, Schuster M, Poglitsch M, Loibner H, Salzberg M, Bruggisser M et al. (2013). Pharmacokinetics and pharmacodynamics of recombinant human angiotensin-converting enzyme 2 in healthy human subjects. Clin Pharmacokinet 52(9): 783-92. doi: 10.1007/s40262-013-0072-7.

Hewitt MG, Miller WT, Reilly TJ, Simpson S (2014). The relative frequencies of causes of widespread ground-glass opacity: a retrospective cohort. Eur J Radiol 83(10): 1970-6. doi: 10.1016/j.ejrad.2014.06.025

Hoffmann M, Kleine-Weber H, Schroeder S, Krüger N, Herrler T, Erichsen S et al (2020).SARS-CoV-2 Cell Entry Depends on ACE2 and TMPRSS2 and Is Blocked by a Clinically Proven Protease Inhibitor. Cell 181(2): 271-280.e8. doi: 10.1016/j.cell.2020.02.052. Epub 2020 Mar 5.

Hooley E, McEwan PA, Emsley J (2007). Molecular modeling of the prekallikrein structure provides insights into high-molecular-weight kininogen binding and zymogen activation. J Thromb Haemost 5(12): 2461. doi: $10.1111 / \mathrm{j} .1538-7836.2007 .02792 . x$

Huang C, Wang Y, Li X, Ren L, Zhao J, Hu Y et al (2020). Clinical features of patients infected with 2019 novel coronavirus in Wuhan, China. Lancet 395(10223): 497-506. doi: 10.1016/S0140-6736(20)30183-5.

Imai Y, Kuba K, Rao S, Huan Y, Guo F, Guan B et al (2005). Angiotensin-converting enzyme 2 protects from severe acute lung failure. Nature 436: 112-6. doi: 10.1038/nature03712. 
Kang MW, Song HJ, Kang SK, Kim Y, Jung SB, Jee S et al (2015). Nafamostat Mesilate Inhibits TNFalpha-Induced Vascular Endothelial Cell Dysfunction by Inhibiting Reactive Oxygen Species Production. Korean J Physiol Pharmacol 19(3): 229-34. doi: 10.4196/kjpp.2015.19.3.229.

Kawase M, Shirato K, van der Hoek L, Taguchi F, Matsuyama S (2012). Simultaneous treatment of human bronchial epithelial cells with serine and cysteine protease inhibitors prevents severe acute respiratory syndrome coronavirus entry. J Virol 86: 6537-6545. doi: 10.1128/JVI.00094-12.

Khan A, Benthin C, Zeno B, Albertson TE, Boyd J, Christie JD et al. (2017). A pilot clinical trial of recombinant human angiotensin-converting enzyme 2 in acute respiratory distress syndrome. Crit Care 21: 234.doi: 10.1186/s13054-017-1823-x

Khan RJ, Jha R, Amera GM, Jain M, Singh E, Pathak A et al (2020). Targeting novel coronavirus 2019: A systematic drug repurposing approach to identify promising inhibitors against 3C-like proteinase and 2'-O-ribose methyltransferase. J Biomol Struct Dyn 1-14. doi: 10.1080/07391102.2020.1753577.

Kim HS, Lee KE, Oh JH, Jung CS, Choi D, Kim Y et al (2016). Cardiac arrest caused by nafamostat mesilate. Kidney Res Clin Pract 35(3): 187-9. doi: 10.1016/j.krcp.2015.10.003.

Kimura S, Tsuji H, Nishimura H, Kato H, Ukimura N, Yano S et al (2002). Bradykinin enhances in vitro procoagulant and antifibrinolytic properties of rat vascular endothelial cells. Thromb Res 106: 41-50. doi: 10.1016/s0049-3848(02)00070-1

Koitka A, Cooper ME, Thomas MC, Tikellis C (2008). Angiotensin converting enzyme 2 in the kidney Clin Exp Pharmacol Physiol 35(4): 420-425. doi: 10.1111/j.1440-1681.2008.04889.x

Kuba K, Imai Y, Rao S, Gao H, Guo F, Guan B et al (2005). A crucial role of angiotensin converting enzyme 2 (ACE2) in SARS coronavirus-induced lung injury. Nat Med 11: 875-879, 2005. doi:10.1038/nm1267.

Kuhr F, Lowry J, Zhang J, Brovkovych V, Skidgel RA (2010). Differential Regulation of Inducible and Endothelial Nitric Oxide Synthase by Kinin B1 and B2 Receptors. Neuropeptides 44(2): 145-54. doi: 10.1016/j.npep.2009.12.004.

Kuznik A, Bencina M, Svajger U, Jeras M, Rozman B, Jerala R (2011). Mechanism of endosomal TLR inhibition by antimalarial drugs and imidazoquinolines. J Immunol 186: 4794-4804. doi: 10.4049/jimmunol.1000702

Leeb-Lundberg LMF (2004). Bradykinin specificity and signaling at GPR100 and B2 kinin receptors. Br J Pharmacol 143(8): 931-932. doi: 10.1038/sj.bjp.0706031.

Letko M, Marzi A, Munster V (2020). Functional assessment of cell entry and receptor usage 348 for SARSCoV-2 and other lineage B betacoronaviruses. Nat Microbiol 5(4): 562-569. doi: 10.1038/s41564-020-0688-y.

Li F (2016). Structure, function, and evolution of coronavirus spike proteins. Annu Rev Virol 3(1): 237-261.

Li F, Li W, Farzan M, Harrison SC (2005a). Structure of SARS coronavirus spike receptor-binding domain complexed with receptor. Science 309(5742): 1864-8. doi: 10.1126/science.1116480

Li WC, Zhang J, Sui JH, Kuhn MJ, Moore S, Luo S et al (2005b). Receptor and viral determinants of SARS-coronavirus adaptation to human ACE2. EMBO J 24(8): 1634-1643. doi: 10.1038/sj.emboj.7600640

Marceau F, Hess JF, Bachvarov DR (1998). The B1 receptors for kinins. Pharmacol Rev 50:357-386.

Maruyama Y, Yoshida H, Uchino S, Yokoyama K, Yamamoto H, Takinami M et al (2011). Nafamostat mesilate as an anticoagulant during continuous veno-venous hemodialysis: a three-year retrospective cohort study. Int J Artif Organs 34(7): 571-6. doi: 10.5301/IJAO.2011.8535

Matsuyama S, Nagata N, Shirato K, Kawase M, Takeda M, Taguchi F (2010). Efficient activation of the severe acute respiratory syndrome coronavirus spike protein by the transmembrane protease TMPRSS2. J Virol 84(24): 12658-12664. https://doi.org/10.1128/JVI.01542-10 
Menachery VD, Yount BL, Josset L, Gralinski LE, Scobey T, Agnihothram S et al (2014). Attenuation and restoration of severe acute respiratory syndrome coronavirusmutant lacking 2'-o-methyltransferase activity. J Virol 88(8): 4251-64. doi: 10.1128/JVI.03571-13.

Milewska A, Zarebski M, Nowak P, Stozek K, Potempa J, Pyrc K (2014). Human coronavirus NL63 utilizes heparan sulfate proteoglycans for attachment to target cells. J Virol 88(22): 13221-30. doi: 10.1128/JVI.0207814.

Monteil V, Kwon H, Prado P, Hagelkrüys A, Wimmer RA, Stahl M et al (2020). Inhibition of SARSCoV-2 Infections in Engineered Human Tissues Using Clinical-Grade Soluble Human ACE2. Cell S00928674(20)30399-8. doi: 10.1016/j.cell.2020.04.004.

Mycroft-West C, Su D, Elli S, Guimond S, Miller G, Turnbull J et al (2020). The 2019 coronavirus (SARSCoV-2) surface protein (Spike) S1 Receptor Binding Domain undergoes conformational change upon heparin binding. bioRxiv. doi: https://doi.org/10.1101/2020.02.29.971093

Ong SWX, Tan YK, Chia PY, Lee TH, Ng OT, Wong MSY et al (2020). Air, surface environmental, and personal protective equipment contamination by severe acute respiratory syndrome coronavirus 2 (SARSCoV-2) from a symptomatic patient. JAMA 323(16):1610-1612. doi: 10.1001/jama.2020.3227.

Ou J, Zhou Z, Zhang J, Lan W, Zhao S, Wu J (2020). RBD mutations from circulating SARSCoV-2 strains enhance the structure stability and infectivity of the spike protein. bioRxiv doi: https://doi.org/10.1101/2020.03.15.991844

Pan PP, Zhan QT, Le F, Zheng YM, Jin F (2013). Angiotensin-Converting Enzymes Play a Dominant Role in Fertility. Int J Mol Sci 14(10): 21071-21086. doi: 10.3390/ijms141021071.

Patel VB, Zhong JC, Grant MB, Oudit GY (2016). Role of the ACE2/angiotensin 1-7 axis of the reninangiotensin system in heart failure. Circ. Res 118: 1313-1326. doi: 10.1161/CIRCRESAHA.116.307708.

Qadri F, Bader M (2018). Kinin B1 receptors as a therapeutic target for inflammation. Expert Opin Ther Targets 22: 31-44. doi: 10.1080/14728222.2018.1409724

Richardson P, Griffin I, Tucker C, Smith D, Oechsle O, Phelan A et al (2020). Baricitinib as Potential Treatment for 2019-nCoV Acute Respiratory Disease. Lancet 395(10223): e30-e31. doi: 10.1016/S01406736(20)30304-4.

Schrezenmeier E, Dörner T (2020). Mechanisms of action of hydroxychloroquine and chloroquine: implications for rheumatology. Nat Rev Rheumatol 16(3): 155-166. doi: 10.1038/s41584-020-0372-x.

Selvarajan S, Lund LR, Takeuchi T, Craik CS, Werb Z (2001). A plasma kallikrein-dependent cascade required for adipocyte differentiation. Nature Cell Biol 3: 267-275. doi: 10.1038/35060059.

Shariat-Madar Z, Schmaier AH (1999). Kininogen-cytokeratin 1 interactions in endothelial cell biology. Trends Cardiovasc Med 9: 238-44.

Shastri MD, Stewart N, Horne J, Peterson GM, Gueven N, Sohal SS et al (2015). In-vitro suppression of IL-6 and IL-8 release from human pulmonary epithelial cells by non-anticoagulant fraction of enoxaparin. PLoS One 10(5): e0126763. doi: 10.1371/journal.pone.0126763

Shi C, Wang C, Wang H, Yang C, Cai F, Zeng F et al (2020). The potential of low molecular weight heparin to mitigate cytokine storm in severe COVID-19 patients: a retrospective clinical study. medRxiv. doi: https://doi.org/10.1101/2020.03.28.20046144.

Shulla A, Heald-Sargent T, Subramanya G, Zhao J, Perlman S, Gallagher T (2011). A Transmembrane serine protease is linked to the severe acute respiratory syndrome coronavirus receptor and activates virus entry. $\mathrm{J}$ Virol 85(2): 873-882. https://doi.org/10.1128/JVI.02062-10 
Sodhi CP, Wohlford-Lenane C, Yamaguchi Y, Prindle T, Fulton WB, Wang S et al (2018). Attenuation of pulmonary ACE2activity impairs inactivation of des-arg9 bradykinin/BKB1R axis and facilitates LPS-induced neutrophil infiltration. Am J Physiol Lung Cell Mol Physiol 314: L17- 31. doi: 10.1152/ajplung.00498.2016.

Tamura Y, Hirado M, Okamura K, Minato Y, Fujii S (1977). Synthetic inhibitors of trypsin, plasmin, kallikrein, thrombin, C1r-, and C1 esterase. Biochim Biophys Acta 484(2): 417-22. doi: 10.1016/00052744(77)90097-3.

Tang N, Bai N, Chen X, Gong J, Li D, Sun Z (2020a). Anticoagulant Treatment Is Associated With Decreased Mortality in Severe Coronavirus Disease 2019 Patients With Coagulopathy. J Thromb Haemost 18(5): 10941099. doi: $10.1111 /$ jth.14817.

Tang N, Li D, Wang X, Sun Z (2020b). Abnormal Coagulation parameters are associated with poor prognosis in patients with novel coronavirus pneumonia. J Thromb Haemost 18: 844-847. doi: 10.1111/jth.14768.

Tay MZ, Poh CM, Rénia L, MacAry PA, Ng LFP (2003). The Trinity of COVID-19: Immunity, Inflammation and Intervention. Nat Rev Immunol. doi: 10.1038/s41577-020-0311-8.

Tipnis SR, Hooper NM, Hyde R, Karran E, Christie G, Turner AJ (2000). A human homolog of angiotensinconverting enzyme. Cloning and functional expression as a captopril-insensitive carboxypeptidase. J Biol Chem 275(43): 33238-33243. doi :10.1074/jbc.M002615200.

Tsai YJ, Hao SP, Chen CL, Lin BJ, Wu WB (2015). Involvement of B2 Receptor in Bradykinin-Induced Proliferation and Proinflammatory Effects in Human Nasal Mucosa-Derived Fibroblasts Isolated from Chronic Rhinosinusitis Patients. PLoS One 10(5): e0126853. doi: 10.1371/journal.pone.0126853.

van den Borne BE, Dijkmans BA, de Rooij HH, le Cessie S, Verweij CL (1997). Chloroquine and hydroxychloroquine equally affect tumor necrosis factor-alpha, interleukin 6 , and interferon-gamma production by peripheral blood mononuclear cells. J Rheumatol 24: 55-60 (1997).

Vickers C, Hales P, Kaushik V, Dick L, Gavin J, Tang J et al (2002). Hydrolysis of biological peptides by human angiotensin-converting enzyme-related carboxypeptidase. J Biol Chem 277: 14838-14843,. doi:10.1074/jbc.M200581200.

Wu F, Zhao S, Yu B, Chen YM, Wang W, Song G et al (2020).. A new coronavirus associated with human respiratory disease in China. Nature 579(7798): 265-269. doi: 10.1038/s41586-020-2008-3.

Xu H, Zhong L, Deng J, Peng J, Dan H, Zeng X (2020a). High expression of ACE2 receptor of 2019-nCoV on the epithelial cells of oral mucosa. Int J Oral Sci 12 (8). https://doi.org/10.1038/s41368-020-0074-x

Xu X, Chen P, Wang J, Feng J, Zhou H, Li X et al (2020b). Evolution of the novel coronavirus from the ongoing Wuhan outbreak and modeling of its spike protein for risk of human transmission. Sci China Life Sci 63(3): 457-460. doi: 10.1007/s11427-020-1637-5

Xu X, Han M, Li T, Sun W, Wang D, Fu B et al (2020c). Effective treatment of severe COVID-19 patients with tocilizumab. Proc Natl Acad Sci U S A 202005615. doi: 10.1073/pnas.2005615117.

Xu Z, Shi L, Wang Y, Zhang J, Huang L, Zhang C et al (2020d). Pathological findings of COVID-19 associated with acute respiratory distress syndrome. Lancet Respir Med 8(4): 420-422. doi: 10.1016/S22132600(20)30076-X

Yamaya M, Shimotai Y, Hatachi Y, Kalonji NL, Tando Y, Kitajima Y et al (2015). The Serine Protease Inhibitor Camostat Inhibits Influenza Virus Replication and Cytokine Production in Primary Cultures of Human Tracheal Epithelial Cells. Pulm Pharmacol Ther 33: 66-74. doi: 10.1016/j.pupt.2015.07.001.

Yuksel M, Okajima K, Uchiba M, Okabe H (2003). Gabexate mesilate, a synthetic protease inhibitor, inhibits lipopolysaccharide-induced tumor necrosis factor-alpha production by inhibiting activation of both nuclear factor-kappaB and activator protein-1 in human monocytes. J Pharmacol Exp Ther 305(1): 298-305. doi: 10.1124/jpet.102.041988. 
Zhang X, Wu J, Du F, Xu H, Sun L, Chen Z et al (2014). The cytosolic DNA sensor cGAS forms an oligomeric complex with DNA and undergoes switch-like conformational changes in the activation loop. Cell Rep 6: 421-430. doi: 10.1016/j.celrep.2014.01.003.

Zhao Y, Zhao Z, Wang Y, Zhou Y, Ma Y, Zuo W (2020). Single-cell RNA expression profiling of ACE2, the receptor of SARS-CoV-2. bioRxiv. doi:https://doi.org/10.1101/2020.01.26.919985.

Zhou F, Yu T, Du R, Fan G, Liu Y, Liu Z et al (2020a). Clinical Course and Risk Factors for Mortality of Adult Inpatients With COVID-19 in Wuhan, China: A Retrospective Cohort Study. Lancet 395(10229): 1054-1062. doi: 10.1016/S0140-6736(20)30566-3.

Zhou J, Fang L, Yang Z, Xu S, Lv M, Sun Z et al (2019). Identification of novel proteolytically inactive mutations in coronavirus 3C-like protease using a combined approach. FASEB J 33(12): 14575-14587. doi: 10.1096/fj.201901624RR

Zhou P, Yang XL, Wang XG, Hu B, Zhang L, Zhang E et al (2020b). A pneumonia outbreak associated with a new coronavirus of probable bat origin. Nature 579(7798): 270-273. doi: 10.1038/s41586-020-2012-7

Zou X, Chen K, Zou J, Han P, Hao J, Han Z (2020). Single-cell RNA-seq data analysis on the receptor ACE2 expression reveals the potential risk of different human organs vulnerable to 2019-nCoV infection. Front Med. doi: 10.1007/s11684-020-0754-0.

\section{Figures and Figure Legends}

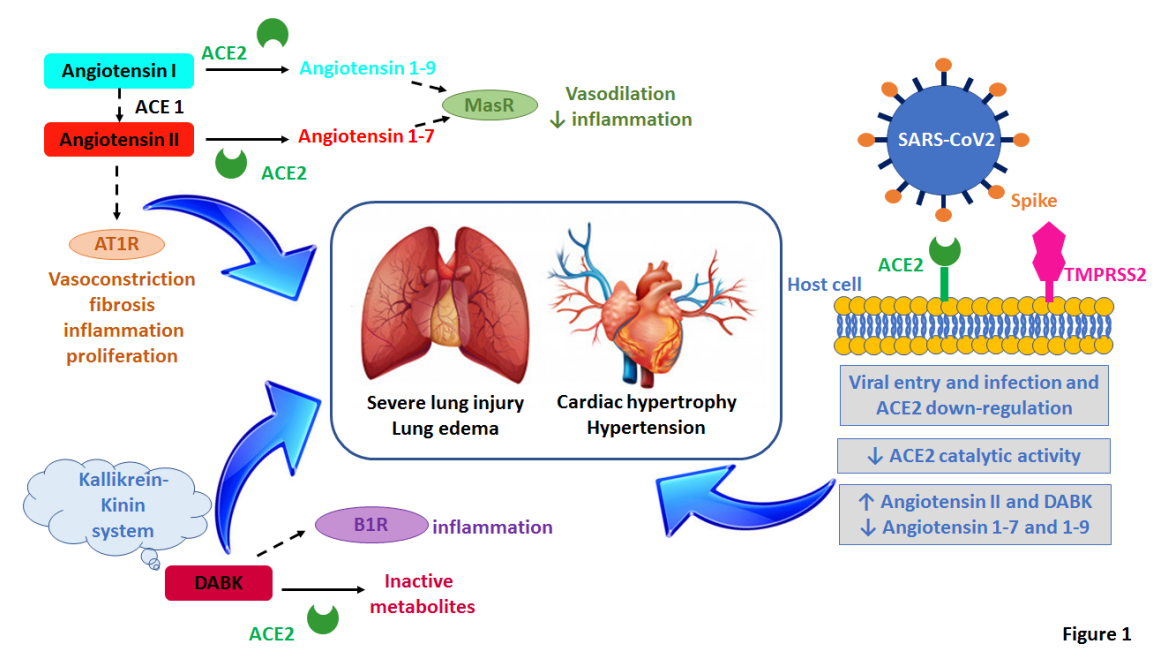

Figure 1. ACE2 function and its regulation in SARS-CoV2 infection.

ACE2 is a carboxypeptidases which catalyzes and inactivates Angiotensin I and Angiotensin II, respectively, into the vasodilator peptides Angiotensin 1-9 and Angiotensin 1-7, which bind Mas receptor (MasR) leading to reduced inflammation and vasodilation. ACE2 also cleaves des-Arg ${ }^{9}$ bradykinin (DABK), a bioactive kinin derived from kininogen pathway, into inactive metabolites. ACE2 is the cell entry receptor for SARS-CoV2; the binding of viral spike glycoprotein with ACE2 and the priming of the spike through the transmembrane protease serine 2 (TMPRSS2), leads to SARS-CoV2 infection. The binding of SARS-CoV2 downregulates ACE2 expression, leading to a reduction of its enzymatic activity and the ensuing increase of Angiotensin II and DABK levels. Angiotensin II takes its deleterious effect by binding the Angiotensin II type 1 receptor (AT1R), whereas DABK concurs to inflammation by binding BK receptor B1 (B1R), resulting in severe lung injury, pulmonary inflammation and edema, increased coagulation, hypertension and cardiac hypertrophy, which are all features of COVID-19 patients. 


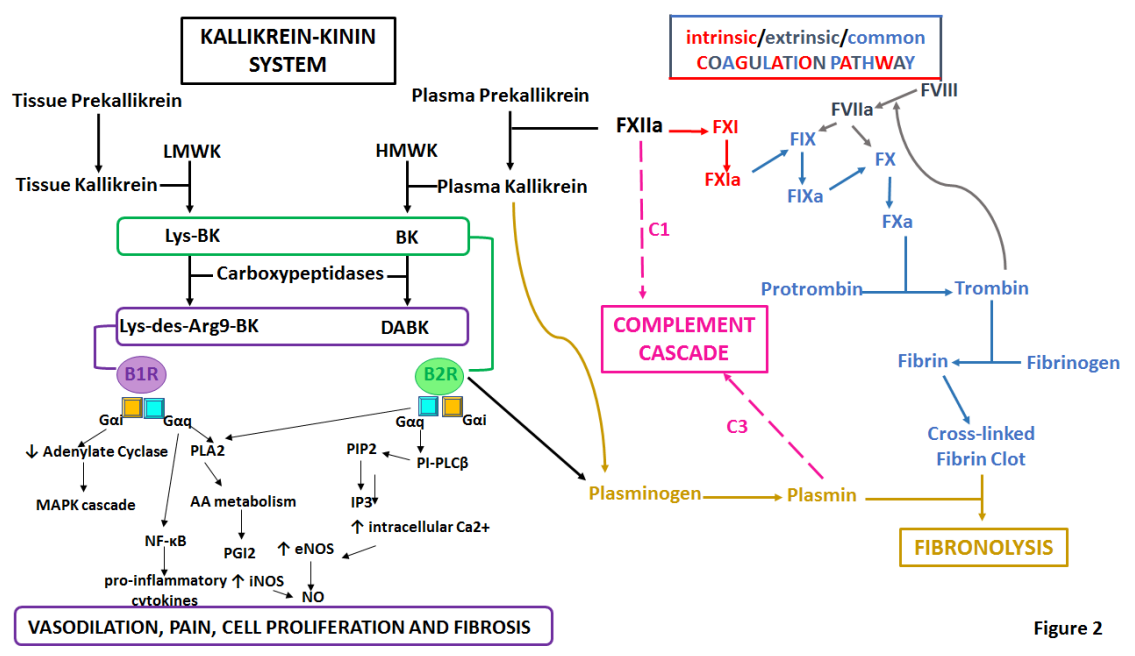

Figure 2. Crosstalk between Kallikrein-Kinin System (KKS), coagulation, fibrinolysis and complement cascade.

Kallikrein-Kinin system (KKS) (black box and arrows) consists of tissue and plasma kallikrein which act on high molecular weight kininogen (HMWK) and low molecular weight kininogen (LMWK) to generate bradykinin (BK) and kallidin (Lys-BK). BK and Lys-BK, and their metabolites (Lys-des-Arg ${ }^{9}$-BK and DABK) act via two G-coupled receptors, B1R and B2R, resulting in increased vascular permeability, vasodilation, edema formation and ultimately hypotension. Plasma kallikrein, which is induced by the reciprocal activation of the Factor XIIa (FXIIa) and plasma prekallikrein, also influences the fibrinolytic pathway by activating plasminogen into plasmin and leading to fibrin degradation and D-dimer generation (yellow box and arrows). Beyond its role in KKS, FXIIa starts the intrinsic coagulation pathway (red arrows). Blood coagulation consists of an intrinsic and extrinsic (grey arrows) pathways, both resulting in activation of Factor $\mathrm{X}$ (FX), which subsequently leads to thrombin and fibrin generation (common pathway; blue arrows). The coagulation cascade is also a starting point for the complement system (pink box and arrows). FXIIa binds C1q component of the complement triggering the classic pathway; moreover, plasmin activation, which is also promoted via B2 signalling, triggers C3 cleavage inducing the activation of both lecithin and extrinsic pathways of the complement.

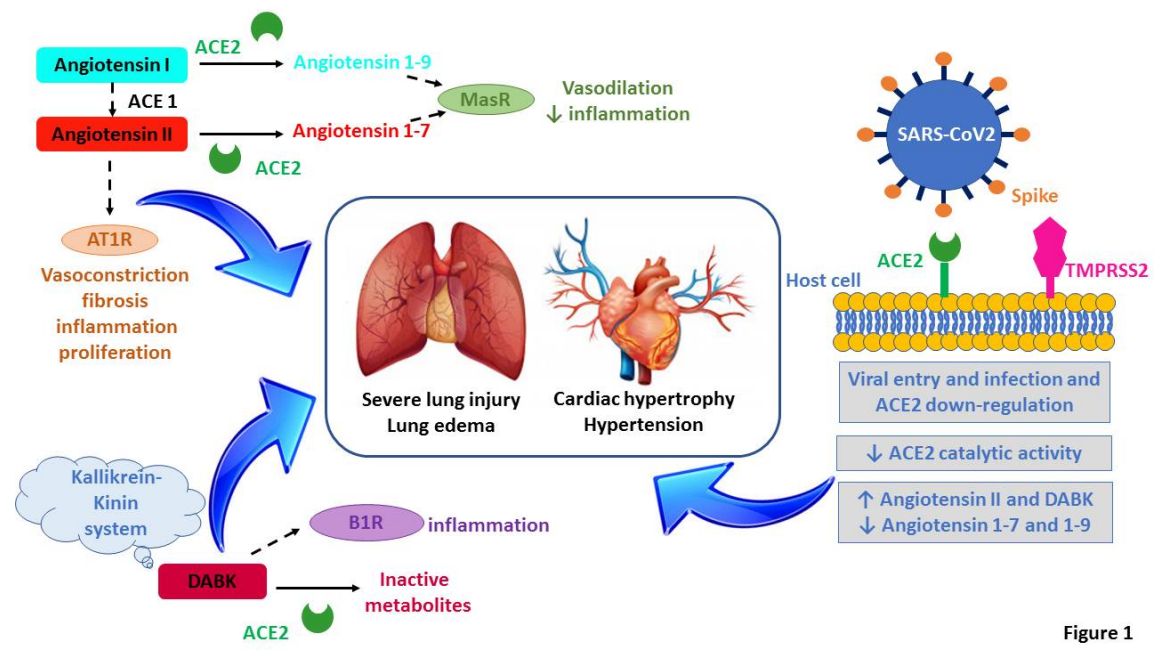




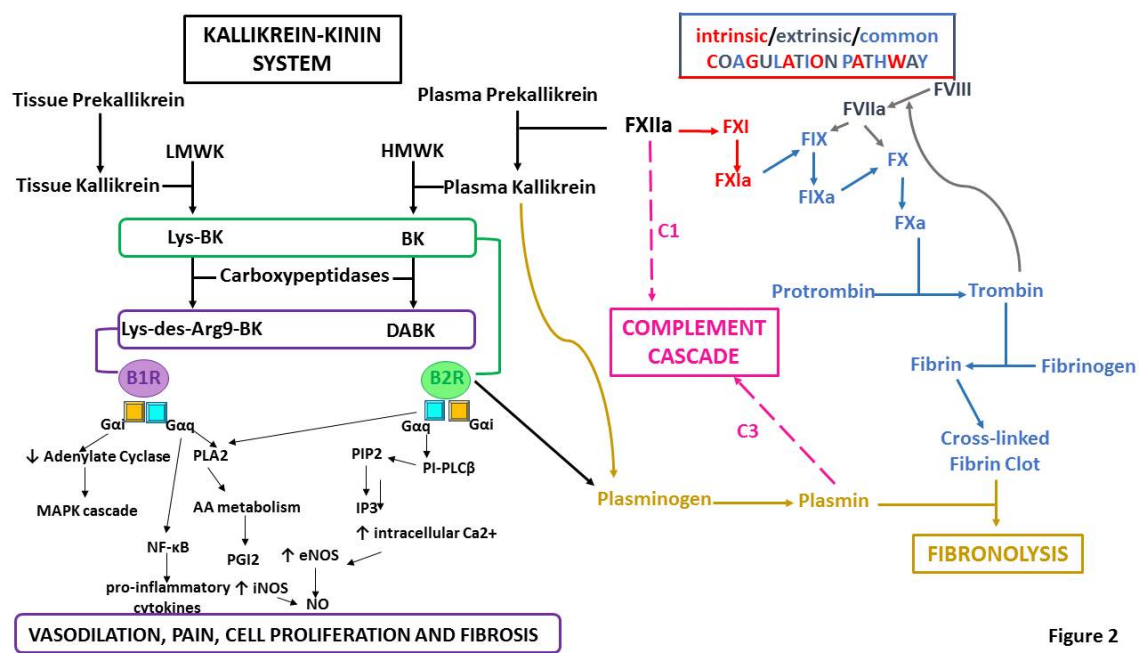

